\title{
SPINT2 controls SARS-CoV-2 viral infection and is associated to disease severity
}

Carlos Ramirez Alvarez ${ }^{1}$, Ashwini Kumar Sharma ${ }^{1}$, Carmon Kee ${ }^{2,3}$, Leonie Thomas ${ }^{1}$, Steeve Boulant $^{2,3}$, Carl Herrmann ${ }^{1, *}$

${ }^{1}$ Health Data Science Unit, Medical Faculty Heidelberg and BioQuant, Heidelberg, Germany; ${ }^{2}$ Department of Infectious Diseases, Virology, Heidelberg University, Germany; ${ }^{3}$ Research Group "Cellular Polarity and Viral Infection", German Cancer Research Center (DKFZ), Heidelberg, Germany *Corresponding author: carl.herrmann@bioquant.uni-heidelberg.de

\section{Abstract}

COVID-19 outbreak is the biggest threat to human health in recent history. Currently, there are over 1.5 million related deaths and 75 million people infected around the world (as of 22/12/2020). The identification of virulence factors which determine disease susceptibility and severity in different cell types remains an essential challenge. The serine protease TMPRSS2 has been shown to be important for S protein priming and viral entry, however, little is known about its regulation. SPINT2 is a member of the family of Kunitz type serine protease inhibitors and has been shown to inhibit TMPRSS2. Here, we explored the existence of a co-regulation between SPINT2/TMPRSS2 and found a tightly regulated protease/inhibitor expression balance across tissues. We found that SPINT2 negatively correlates with SARS-CoV-2 expression in Calu-3 and Caco-2 cell lines and was down-regulated in secretory cells from COVID-19 patients. We validated our findings using Calu- 3 cell lines and observed a strong increase in viral load after SPINT2 knockdown. Additionally, we evaluated the expression of SPINT2 in datasets from comorbid diseases using bulk and scRNA-seq data. We observed its down-regulation in colon, kidney and liver tumors as well as in alpha pancreatic islets cells from diabetes Type 2 patients, which could have implications for the observed comorbidities in COVID-19 patients suffering from chronic diseases.

\section{Introduction}

SARS-CoV-2 entry requires a two-step process: first, the envelope protein spike (S) binds to the viral cellular receptor Angiotensin-converting enzyme 2 (ACE2) membrane protein ${ }^{1}$ and is then proteolytically activated by cellular serine proteases like TMPRSS2, TMPRSS4 and Furin ${ }^{2-4}$. TMPRSS2 has been proposed as a putative drug target ${ }^{3,5,6}$ and as a biomarker for COVID19 disease severity ${ }^{7,8}$. Despite its central role, the regulation of TMPRSS2 is poorly understood, although its activation by androgen response elements has been documented in normal and tumor prostate tissues ${ }^{9}$. 
SPINT2, a member of the Kunitz-type serine proteases inhibitors ${ }^{10}$ has been shown to inhibit TMPRSS2 protease activity 11,12 which could have implications for COVID-19 disease. A down-regulation of SPINT1, SPINT2 and SERPINA1 has been reported in colorectal Caco-2 cells infected with SARS-CoV-2 ${ }^{13}$, however, a clear association between SPINT2 activation and viral permissivity has not been confirmed. To fill this gap, we evaluated the existence of coregulation between SPINT2/TMPRSS2 and found common transcription factors (TF) associated with genomic loci for both genes which was also in line with a consistent correlation of the genes across cell types. This coregulation suggested a modulation of SARS-CoV-2 infection by SPINT2. We could corroborate a negative correlation between SPINT2 gene expression and SARS-CoV-2 viral load in Calu-3 and Caco-2 cell lines. To validate our findings we knocked-down SPINT2 in Calu-3 and observed an increase in the number of SARS-CoV-2 infected cells. We hypothesized that SPINT2 levels would be lower in SARS-CoV-2 target cells from COVID-19 patients with severe symptoms which we could indeed observe in secretory cells from nasopharynx samples. This suggests that SPINT2 can be used as a biomarker for disease susceptibility. Finally, it is known that SPINT2 is down-regulated among different types of tumors ${ }^{14}$ and we were able to corroborate this by systematically evaluating bulk- and scRNA-seq datasets which suggest a possible association to the COVID-19 comorbidity observed in cancer patients.

\section{Results and Discussions}

\section{SPINT2 and TMPRSS2 are coregulated across tissues}

TMPRSS2 proteolytic activity inhibition by SPINT2 has been previously reported ${ }^{11,12}$. We investigated a coregulation between SPINT2 and TMPRSS2, as a similar shared regulation through the transcription factor (TF) CDX2 has been described for SPINT1 and ST14 (Matriptase) previously in enterocytes ${ }^{15}$. Since SPINT2 is also able to regulate ST14 activity in small and large intestines ${ }^{16}$ we decided to use enterocytes as a model to test this hypothesis. In order to find common TFs regulators of SPINT2/TMPRSS2 we performed two independent analysis: i) A footprinting analysis of chromatin open regions using ATAC-seq data from Human Intestinal Organoids ${ }^{17}$ identifying potential TF binding sites and ii) Using scRNA-seq data from ileum derived organoids ${ }^{18}$ we calculated the activity of transcription factors based on the gene expression of their targets using the SCENIC algorithm ${ }^{19}$. TF activities were then correlated to SPINT2 and TMPRSS2 gene expression. We identified common TFs inferred to be bound to the open chromatin sites in the SPINT2 and TMPRSS2 genomic loci (Figure 1A, top and bottom, respectively) and those with TF activities positively correlated to both SPINT2 and TMPRSS2 gene expression (Figure 1B, top right quadrant). Comparing these two sets of TFs, we found ten shared regulators: ELF3, FOS, FOSL1, FOXC1, IRF1, IRF7, JUND, JUNB, ONECUT3 and KLF4. Interestingly, many of these regulators play a role in immune response upon infection, suggesting a possible feedback mechanism. SARS-CoV-2 infection 
has been shown to upregulate FOS expression in Huh7.5 and A549 cell lines ${ }^{20}$. IRF1 and IRF7 are interferon regulatory factors which regulate infection responses, and have been observed to be upregulated in COVID-19 patients ${ }^{21}$. JUNB has been found in SARS-CoV-2 infection gene expression signatures in Calu-3 and Caco-2 cell lines ${ }^{22}$. ELF3 is an important factor controlling the development of epithelium tissues ${ }^{23}$ including intestinal epithelia ${ }^{24}$. Based on our analysis, we depict the regulatory model in which SPINT2 and TMPRSS2 gene expression are coregulated by common TFs in ileum enterocytes, hence maintaining the protease/inhibitor balance (Figure 1C). On the other hand, down-regulation of TMPRSS2 enzymatic activity by SPINT2 possibly maintains viral load at a low level. In line with this, we observed that the expression of SPINT2 and TMPRSS2 are positively correlated across normal human tissues (Figure 1D). Interestingly, tissues which have been shown to be targets for the virus display the highest correlation between SPINT2 and TMPRSS2 expression. Also, at single cell resolution, we observed that both genes are specifically co-expressed in many cell types ${ }^{25}$ (Figure 1E) which corroborates the inferred coregulation.

\section{Deriving a SARS-CoV-2 permissivity signature}

Given the previous observed association between SPINT2 and TMPRSS2, a major SARS-CoV-2 virulence factor ${ }^{3}$, we asked whether SPINT2 could account for differences in viral permissivity. Calu-3 and $\mathrm{H} 1299$ cells have been previously reported as SARS-CoV-2 permissive and non-permissive cell lines, respectively ${ }^{22}$. To that end, we inferred a SARS-CoV-2 permissivity signature using the following approach: we calculated Differentially Expressed Genes (DEGs) between non-infected Calu-3 and H1299 cells (set $z$ in Figure 2A). Then, from this list we excluded any genes that might be related to immune responses, even in mock-infected cells. In order to do so, we calculated DEGs between the infected vs mock-infected cells from both Calu- $3(x)$ and $\mathrm{H} 1299$ cell lines $(y)$ and then filtered out these genes from $z$ to obtain non-viral inducible genes (i') (Figure 2A). Using this approach, we identified a set of 480 candidate genes which might contribute to infection permissivity (see Supplementary Table 1). We used normalized expression values for these 480 genes as input to train a Random Forest (RF) model for predicting the cumulative sum of viral gene expression in Calu-3 infected cells (Figure 2B). We then used the top 25\% ranked genes for further analysis. SPINT2 was found among the top ranked genes (Figure 2B). Of the top ranked genes, 21 corresponded to genes with functional annotations related to viral infection (Figure 2B inset pie chart, Supplementary Table 1), four have been reported to participate and/or interact directly with SARS-CoV-2 ${ }^{26}$, eight corresponded to curated receptors ${ }^{27}$ or ligands in the CellPhone Data Base ${ }^{28}$ and 13 are cell membrane surface proteins. Importantly, by intersecting the set of genes in the permissivity signature with a reported SARS-CoV-2 viral infection transcriptional signature ${ }^{29}$, we only found 3 hits (CXCL5, LGALS3BP and EHF), confirming that most of the identified genes in our permissivity signature are not viral-induced, but likely represent a priori susceptibility factors. We found the following Heat Shock Proteins (HSP): HSPB1, HSPA8 and HSPD1 to be differentially expressed. In a previous study a different HSP, HSP90, was observed to correlate to SARS-CoV-2 
viral load in Calu-3 cells and its inhibition reduced viral infection ${ }^{22}$. We also found several ribosomal proteins (RPL9, RPL23, RPL26, RPL28, RPL38, RPS7, RPS12 and RPS27A) and elongation factors (EIF3A, EIF4A2 and EIF4B) which could be related to viral protein translation and ER stress response 30. In order to confirm that the permissivity signature are not just reflecting tissue specific or immune signatures, a Pathway Enrichment Analysis (PEA) was performed using the top ranked genes. Interestingly, we found an enrichment of host-viral interactions processes, protein stabilization and Endoplasmic Reticulum (ER) trafficking pathways (Figure 2C).

Next, we investigated if these susceptibility genes, identified from lung-derived cell lines, are also expressed in other cell types. Therefore, we ranked the cell types in the Human Cell Landscape dataset $(\mathrm{HCL})^{25}$ based on the permissivity score derived from our top ranked genes in the permissivity signature (Figure 2D) and found that stratified epithelial, basal, AT2 lung cells and enterocytes were among the top-ranked cell types which correspond to cell types known to be infected by the virus ${ }^{31,32}$.

In order to further refine our permissivity signature by going beyond transcriptional levels, we used protein expression levels of a previously released proteomic dataset from SARS-CoV-2 infected Caco-2 cells ${ }^{13}$. We determined the Spearman correlations of the translation rates for the top ranked genes to that of the $\mathrm{N}$ and $\mathrm{S}$ viral proteins (Figure $2 \mathrm{E}$ ). Some of the highly correlated genes (both negative and positive) have been previously reported to participate in viral infection processes. For example, LGALS3BP is a glycoprotein secreted molecule with antiviral properties observed in HIV and Hantavirus infection ${ }^{33,34}$ and in the regulation of LPS induced endotoxin shock in murine models ${ }^{35}$. CLIC1 has been previously identified as a virulence factor of Merker Cell Polyomavirus (MCPyV) which is upregulated during infection and promotes the development of Merkel Cell Carcinoma ${ }^{36}$. HSPD1 has been shown to promote viral infection of HIV, HBV and Influenza viruses ${ }^{37}$. Interestingly, SPINT2 was consistently correlated to viral translation (Figure 2E). Furthermore, the correlation of SPINT2 with viral gene expression is negative and this trend is consistent in both Caco-2 and Calu-3 cell lines, indicating a repressive role on SARS-CoV-2 infection (Supplementary Figure $1 \mathrm{~A}$ and $\mathbf{B})$. Hence, these findings suggest that SPINT2 represents a permissivity factor that negatively correlates with SARS-CoV-2 infection.

\section{SPINT2 knockdown increases viral load in Calu-3 cells}

To experimentally validate the negative correlation of SPINT2 expression with SARS-CoV-2 viral gene expression, we hypothesized that this gene could have a direct influence on SARS-CoV-2 infection by impairing early steps of viral entry. Hence, to test our hypothesis, we knocked-down SPINT2 using small-hairpins RNA in the human lung carcinoma derived line Calu-3 cells. SPINT2 expression was readily detectable in wild-type (WT) Calu-3 cells. When Calu-3 cells were transduced with a specific shRNA directed against SPINT2, SPINT2 levels were significantly decreased compared to WT cells or cells transduced with a scrambled shRNA (Figure 3A). To address the impact of SPINT2 knocked-down on the permissivity of Calu-3 cells to SARS-CoV-2, WT, scrambled and SPINT2 knocked-down cells were infected with SARS-CoV-2 using the same multiplicity of infection (MOI). 
Knocked-down of SPINT2 resulted in an almost two-fold increase in the number of cells positive for SARS-CoV-2 (Figure 3B-C). In order to test the hypothesis whether SPINT2 could modulate viral load by regulating TMPRSS2 expression, we monitored its fold change expression. Interestingly, TMPRSS2 gene expression was found to be higher in SPINT2 knocked-down cells when compared to WT or scramble cells (Figure 3D). Together, our results show that genetic depletion of SPINT2 results in an increased susceptibility of Calu-3 cells to SARS-CoV-2 infection, which is in agreement with our analysis suggesting that SPINT2 expression negatively correlates with infection.

SPINT2 is negatively correlated to viral load and is down-regulated in severe COVID-19 cases

Given the observed negative correlation between SPINT2 expression and SARS-CoV-2 infection in cell lines (Figure 2E, Supplementary Figure 1A, B) we next investigated if SPINT2 expression is associated with disease severity in COVID-19 patients. We used a publicly available scRNA-seq dataset on nasopharynx swabs samples from patients with severe and mild symptoms ${ }^{38}$. We correlated a list of serine proteases and inhibitors (SPRGs, Supplementary Table 2) to the viral RNA reads and found that SPINT2 was the second most negatively correlated gene (Figure 4A). Then, we selected the cell cluster with the highest expression of SPINT2, which correspond to secretory cells (Supplementary Figure 2A) and among these cells, observed a lower SPINT2 gene expression in cells from critical COVID19 cases compared to moderate cases (Figure 4B). This finding is particularly relevant since secretory cells are primary targets of viral infection ${ }^{39}$. We also evaluated data on Peripheral Blood Mononuclear Cells (PBMC) from severe COVID-19 patients ${ }^{40}$. In this dataset, SPINT2 was found to be strongly expressed in Dendritic Cells (DC), plasmacytoid DC (pDC), and stem cells (SC) and eosinophils (Supplementary Figure 2B). Among these cells, again, we observed lower SPINT2 expression in patients from Intensive Care Units (ICU) (Figure 4C). Additionally, we could also corroborate the negative correlation of SPINT2 and viral load using bulk RNA-seq data from lung autopsies of COVID-19 deceased patients ${ }^{41}$. We calculated the correlations of gene expression between SPINT2, ACE2 and TMPRSS2 to E, M, N and S viral genes and observed the similar negative correlation (Supplementary Figure 2C). Collectively, this evidence suggests that SPINT2 expression level could be associated to COVID-19 disease severity.

SPINT2 is down-regulated in multiple tumor types and pancreatic cells from T2D patients

COVID-19 patients with previous records of chronic diseases like cancer or diabetes are considered at higher risk ${ }^{42-46}$. Also, SPINT2 gene silencing by promoter hypermethylation has been reported in multiple tumor types which promotes tumor progression ${ }^{14,47-49}$. For this reason, we hypothesized that SPINT2 down-regulation in tumor cells would increase viral infection permissivity which among others, could be one of the mechanisms behind the comorbidity observed in COVID-19 patients. We screened lung, colon, liver and hepatic tumor datasets to evaluate the differences in SPINT2 gene 
expression between tumor and paired normal samples. We found statistically significant down-regulation of SPINT2 in the kidneys and liver tumors (Supplementary Figure 3A). Similarly, using comparable tumor scRNA-seq datasets ${ }^{50-54}$ we observed a down-regulation of SPINT2 in colon adenocarcinoma (epithelial cells), renal clear cell carcinoma (endothelial cells) and hepatocellular carcinoma (hepatocytes) (Figure 5). Interestingly, we were able to detect SPINT2 down-regulation in colorectal tumor epithelial cells at single cell level but not in bulk RNA-seq data suggesting that SPINT2 expression might me modulated in specific cell subtypes (Figure 5 and Supplementary Figure 4A). In lung adenocarcinomas, we found SPINT2 upregulation in tumors both in TCGA and scRNA-seq data which might reflect the existence of different determinants for comorbidity in lung tissues independent of SPINT2 modulation. We also looked at the expression of SPINT2 in pancreatic cells from diabetes type 2 (DT2) patients ${ }^{54}$. Islet cells have high SPINT2 expression when compared to other cell types like endothelial cells (see Supplementary Figure 4B). We observed a strong down-regulation of SPINT2 in alpha-cells of DT2 patients, which have been shown to be primary targets of the SARS-CoV-2 virus. This down-regulation of the virulence associated factor SPINT2 might contribute to the comorbidity between COVD19 and DT2.

\section{Discussion}

In this study, we describe a tight protease-inhibitor/protease balance at the gene expression level between SPINT2 and TMPRSS2, a major co-receptor of SARS-CoV-2. We found Transcription Factor Binding Sites (TFBS) for ten regulators including IRF1, IRF3, JUNB, JUND and ELF3 whose TF activities were found to be correlated to both SPINT2 and TMPRSS2 gene expression which suggests their possible role as common regulators of both genes. Interestingly, ELF3 and IRF7 TF activity has been found to be modulated in SARS-CoV-2 infected vs bystander enterocytes from ileum ${ }^{18}$, which could point to viral load modulation mediated by TMPRSS2 and SPINT2 through these TFs. We show that SPINT2 and TMPRSS2 gene expression levels are correlated across cell types and tissues. Interestingly, known SARS-CoV-2 target tissues have high correlation values and co-expression for both genes which suggest that SPINT2 could play a role in SARS-CoV-2 viral entry.

Currently, it is unclear what are the molecular signatures that determine viral permissivity and how they are related to disease severity. We inferred a SARS-CoV-2 permissivity signature, using differentially expressed genes between permissive and non-permissive cell lines from which we removed viral induced genes. We were able to find SPINT2 in this permissivity signature and observed a negative correlation to SARS-CoV-2 viral load in Calu-3 cells. We also corroborated this trend at the protein level in Caco-2 cells. During the preparation of these manuscript, a study from Bojkova D et al, 2020 was published suggesting a possible role of SPINT1, SPINT2 and SERPINA1 in viral infection by observing the down-regulation of their protein levels in infected cells and also by evaluating the effect of Aprotinin a non-specific SP inhibitor on viral load ${ }^{6}$. However, here for the first time by knocking down SPINT2, we provide a direct causal evidence that SPINT2 is indeed able to modulate SARS-CoV-2 infection. 
SPINT2 inhibits TMPRSS2 enzymatic activity through its KD1 and KD2 domains ${ }^{12}$. Interestingly, we could observe an up-regulation of TMPRSS2 mRNA expression in the SPINT2 knocked-down Calu-3 cells. Further investigation is needed to explore this regulation at the gene expression level. Although, it has been reported previously that SPINT2 can modulate ST14 protein activity by regulating its shedding from the cell membrane of mouse intestinal epithelial cells ${ }^{16}$ which suggest that SPINT2 could modulate serine proteases activity through different mechanisms. Interestingly, SPINT2 has been reported to regulate transcription of certain genes like $C D K 1 A$ via histone methylation ${ }^{55}$. Our findings suggest that SPINT2 regulates SARS-CoV-2 viral infection through the inhibition of TMPRSS2, however, we cannot discard the possibility of an indirect interaction.

We found a lower expression of SPINT2 in secretory cells from COVID-19 patients with severe symptoms ${ }^{38}$. This could have implications for COVID-19 disease severity since secretory cells have been shown to be the target of SARS-CoV viral infection using organotypic human airway epithelial cultures ${ }^{39}$. We found SPINT2 in the permissivity signature from which we filtered out viral induced genes, suggesting that this gene could be used as a marker for predicting COVID-19 disease susceptibility prior to infection, however this needs to be further evaluated.

Serine proteases (SPS) have been reported to be abnormally regulated in diverse chronic diseases ${ }^{14,56-58}$. For example, during carcinogenic development SPs influence metastasis and cancer progression ${ }^{59,60}$, while in the context of diabetes they control fibrinolysis, coagulation and inflammation which in turn affects disease severity ${ }^{57}$. This led us to hypothesize that shared molecular mechanisms between some chronic diseases and COVID-19 could be explained in part by the regulation of SPINT2. We observed SPINT2 down-regulation in Hepatocellular Carcinoma (HCC), Colon Adenocarcinoma (COAD) and renal Clear Cell Carcinoma (rCCC) tumor cells. SPINT2 down-regulation in liver has been reported to contribute to the development of HCC by the binding and inhibition of the serine protease HGFA which transforms Hepatocyte Growth Factor (HGF) into its active form which in turn promotes metastasis, cell growth and angiogenesis ${ }^{14,61}$ and the same mechanism has been suggested for $\mathrm{rCCC}^{9}$. A marked down-regulation of SPINT2 can be observed in alpha islets pancreatic cells from diabetes patients. It has been reported that islet cells can be infected by SARS-CoV-2 which could contribute to the onset of acute diabetes ${ }^{62}$. Hence, these results suggests that kidney, colon and liver tumor types as well as pancreatic islets cells from diabetic patients could be more permissive and susceptible to SARS-CoV-2 viral infection due to an imbalance of SPINT2 gene expression, which could lead to the disruption of the protease-inhibitor/protease balance ${ }^{63}$.

In conclusion, we showed for the first time that SPINT2 is a permissivity factor that modulates SARS-CoV-2 infection. This modulation could be explained by the balance of TMPRSS2/SPINT2 (serine protease/inhibitor) that we observed at the gene expression level across several tissues. We also found lower SPINT2 gene expression in samples from COVID-19 patients with severe symptoms, hence, this gene might represent a biomarker for predicting disease severity. We also found SPINT2 down-regulation in tumor types which could have implications for the observed comorbidities in COVID-19 patients with cancer. 


\section{Methods}

\section{Cell line and Viruses}

Human lung adenocarcinoma cell lines Calu-3 (ATCC HTB-55) were cultured in Dulbecco's modified Eagle's medium (DMEM) supplemented with Glutamax (Gibco), 10\% fetal bovine serum and 1\% penicillin/streptomycin while Vero E6 cells (ATCC CRL 1586) were cultured in Dulbecco's modified Eagle's medium supplemented with $10 \%$ fetal bovine serum and $1 \%$ penicillin/streptomycin (Gibco). Calu-3 cell lines stably expressing the SPINT2 knockdown were generated by lentiviral transduction.

Production of lentiviral constructs expressing shRNA against SPINT2

Oligonucleotides encoding the sequence for SPINT2 knockdown were designed from the TRC library based on Genetic Perturbation Platform (GPP) Web Portal, cloneID: TRCN0000073581 (Table 1) ${ }^{64}$ Annealed oligonucleotides were ligated with the Agel-HF and EcoRI-HF digested pLKO.1 puro vector (Add gene \#8453) using the T4 DNA Ligase (New England Biolabs) and the resulting plasmids were transformed into E. coli DH5a-competent cells. Amplified plasmid DNA was purified using the NucleoBondR PC 100 kit by Marchery-Nagel following the manufacturer's instructions.

Table 1: Oligonucleotides for shRNA expression. Bold characters mark the respective target sequence.

\begin{tabular}{|l|l|}
\hline Name & Sequence \\
\hline shSPINT & CCGGCAGCTGGTGAAGAACACATATCTCGAGATATGTGTTCTTCACCAGCTGTTT \\
$2 \mathrm{fw}$ & TTG \\
\hline shSPINT & AATTCAAAAACAGCTGGTGAAGAACACATATCTCGAGATATGTGTTCTTCACCAG \\
$2 \mathrm{rev}$ & CTG \\
\hline $\begin{array}{l}\text { shScram } \\
\text { bled fw }\end{array}$ & CAACAAGATGAAGAGCACCAACTCGAGTTGGTGCTCTTCATCTTGTTGTTTT \\
\hline $\begin{array}{l}\text { shScram } \\
\text { bled rev }\end{array}$ & AAAACAACAAGATGAAGAGCACCAACTCGAGTTGGTGCTCTTCATCTTGTTG \\
\hline
\end{tabular}

Lentivirus production and selection of stable cell lines

HEK293T cells (ATCC CRL-3216) were seeded on $10 \mathrm{~cm}^{2}$ dishes and allowed to adhere for 36 hours. The cells were transfected with $4 \mu \mathrm{g}$ of pMD2.G (Addgene \#12259), $4 \mu \mathrm{g}$ of psPAX2 (Addgene $\# 12260$ ) and $8 \mu \mathrm{g}$ of purified pLKO.1 plasmid containing the shRNA constructs upon reaching $70 \%$ confluency. Cell supernatant containing lentivirus was harvested $72 \mathrm{~h}$ post-transfection, filtered through a $45 \mu \mathrm{M}$ Millex HA-filter (Merck Millipore) and purified by ultracentrifugation at $27,000 \times \mathrm{g}$ for $90 \mathrm{~min} .2 \times 10^{5}$ Calu- 3 cells were seeded onto collagen coated 6 -well plates $24 \mathrm{~h}$ prior to transduction. Cell medium was replaced with $3 \mathrm{~mL}$ medium containing $20 \mu \mathrm{L}$ of the purified lentivirus and $3 \mu \mathrm{l}$ 
bioRxiv preprint doi: https://doi.org/10.1101/2020.12.28.424029; this version posted December 28, 2020. The copyright holder for this preprint (which was not certified by peer review) is the author/funder, who has granted bioRxiv a license to display the preprint in perpetuity. It is made available under aCC-BY-NC-ND 4.0 International license.

polybrene transfection reagent (Merck Millipore). Medium was supplemented with $10 \mu \mathrm{g} / \mathrm{mL}$ puromycin for selection of successfully transduced cells two to three days after transduction.

\section{SARS-CoV-2 viral infection}

The SARS-CoV-2 isolate used in the experiments was obtained from the swab of a SARS-CoV-2 positive patient from the Heidelberg University Hospital. The virus was isolated and propagated in Vero E6 cells. All SARS-CoV-2 infections were performed with a multiplicity of infection of 0.04 as determined in Vero E6 cells. Prior to infection, culture media was removed and virus was added to cells and incubated for 1 hour at $37^{\circ} \mathrm{C}$. Fresh media was added back to the cells upon virus removal.

RNA isolation, $c D N A$ synthesis and $q P C R$

Cells were harvested 24 hours post infection for RNA isolation using RNAeasy RNA extraction kit (Qiagen) as per manufacturer's instructions. Complementary DNA was synthesized using iSCRIPT reverse transcriptase (BioRad) from $250 \mathrm{ng}$ of total RNA per $20 \mu \mathrm{L}$ reaction according to the manufacturer's instructions. Quantitative RT-PCR assay was performed using iTaq SYBR green (BioRad) as per manufacturer's instructions. The expression of target genes was normalized to endogenous control TBP. Primer sequences are as below:

\begin{tabular}{|l|l|l|l|}
\hline Target gene & Species name & Forward sequence & Reverse sequence \\
\hline TBP & Human & ccactcacagactctcacaac & ctgcggtacaatcccagaact \\
\hline COV21 & SARS-CoV-2 & gcctcttctgttcctcatcac & agacagcatcaccgccattg \\
\hline SPINT2 & Human & attgcctctttctcatcac & gactcccataaagaaagcac \\
\hline TMPRSS2 & Human & acctgatcacaccagccatg & cttcgaagtgaccagaggcc \\
\hline
\end{tabular}

The fold change in SARS-CoV-2 genome copy number was calculated using input as a reference. Input samples were harvested directly post-infection and accounted for the basal viral genome copy number detected due to viruses attaching to the cell membrane.

\section{Indirect Immunofluorescence Assay}

Cells were seeded on iBIDI glass bottom 8-well chamber slides which are previously coated with $2.5 \%$ human collagen in water for at least 1 hour. Cells were fixed in $4 \%$ paraformaldehyde (PFA) for 20 mins at room temperature (RT) 24 hours post infection. Cells were washed in 1X PBS and permeabilized in $0.5 \%$ Triton-X for 15 mins at RT. 30 minutes of blocking were carried out using $3 \%$ BSA-PBS at RT. Mouse monoclonal antibody against SARS-CoV-2 Nucleocapsid (NC) protein (Sino biologicals MM05) as primary antibody was diluted in 1\% BSA-phosphate-buffered saline (PBS) and incubated for $1 \mathrm{~h}$ at RT. Cells were washed with $1 \mathrm{X}$ PBS three times and incubated with secondary antibodies conjugated with AF488 (Molecular Probes) and DAPI for 30-45 mins at RT. Cells were washed in $1 \mathrm{X}$ PBS three times and maintained in PBS. Cells were imaged on a Nikon/Andor Spinning Disc Confocal microscope to quantify the number of infected cells relative to the number of nuclei. 


\section{Statistics and computational analyses and statistics}

In order to quantify infected cells from indirect immunofluorescent stained samples, ilastik 1.2.0 was used on DAPI images to generate a mask representing each nucleus as an individual object. These masks were used on CellProfiler 3.1.9 to measure the intensity of the conjugated secondary antibodies in each nucleus. A threshold was set based on the basal fluorescence of non infected samples, and all nuclei with a higher fluorescence were considered infected cells.

\section{Calu-3 and 1299 cells preprocessing}

For Calu- 3 cells we filter out cells with an extremely high number of detected genes $(>50,000)$ which probably corresponds to doublets. In H1299, since few cells were detected to be infected, because this line is non-permissive, in order to obtain DEGs we defined infected cells as those with cumulative sum of viral genes expression $>0$.

\section{Assessing non-viral induced permissivity signatures}

As we wanted to differentiate between permissivity and infection signatures, we first looked for differentially expressed genes in SARS-CoV-2 permissive vs non-permissive cell lines and then we removed all the genes which were up- or down-regulated during infection (Figure 2A). We performed Differential Expression Analysis using Seurat ${ }^{65}$ (DEA) between Calu-3 and $\mathrm{H} 1299$ cells in non-infected mock cells at 4 hours of culture $(z)$. Then, we obtained DEGs of Calu-3 infected vs mock at 12 hours post infection ( $x$ ); we did the same with $\mathrm{H} 1299$ infected cells vs mock-infected cells at 4 hpi $(y)$ In all DEA we set a Log Fold Change $(F C)=0.25$ threshold. Finally, we removed these infection signatures from the DEGs of Calu-3 vs $\mathrm{H} 1299$ to obtain the permissivity gene signature (i').

\section{Ranking genes using RF and Pathway Enrichment Analysis}

A Random Forest (RF) regression analysis was performed using the normalized gene expression of the permissivity signature to predict the cumulative sum of the expression of viral genes in Calu-3 cells at $12 \mathrm{hpi}$. We trained the RF using a random subsample of $75 \%$ and tested the results with the remaining set. Next, we estimated the feature importance for each of the permissivity signature genes and performed enrichment analysis using enrichR ${ }^{66}$ on the top $25 \%$ ranked genes.

\section{Scoring permissivity signatures}

For the scoring of cells based on the permissivity signature among cell types in the HCL dataset, we used the top $25 \%$ RF ranked genes and applied the AddModuleScore function of Seurat setting nbin $=100$.

\section{SPINT2 expression correlation to viral gene expression}

For the translatome correlation analysis, the summed intensity normalized values were used as provided in the study ${ }^{13}$. In order to compute the correlations of SPRGs (Supplementary Table 2) to the viral reads in the scRNA-seq data from Chua RL et al, 2020 the raw count matrices were 
extracted from the Seurat object provided by the authors, splitted by sample and then imputed using scimpute ${ }^{67}$ with the following parameters: drop_thr=0.5 and Kcluster equal to the number of annotated cell types in each matrix. The imputed matrices were then merged and log2 normalized. Finally, correlations were performed restricted to infected cells (viral read counts $>0$ ). In the bulk RNA-seq data from deceased COVID-19 patients log2 RPM of normalized counts are used. In both cases correlation to viral genes were carried out using spearman coefficients.

scRNA-seq data preprocessing

In order to have a standardized workflow for the processing of SCRNA-seq data we used SCT normalization using the Seurat workflow for every dataset except for Human Cell Landscape data where log2 normalization and scaling were performed since this dataset is large and using SCT was unpractical. HCC data were downloaded from GEO (GSE149614) and reprocessed. We used the Louvain method implemented in Seurat for community detection and clusters were identified by using tissue markers. We used the markers used to characterize cell types from an independent scRNA-seq human liver atlas ${ }^{68}$ and using these markers identified clusters of epithelial, endothelial, hepatocytes, Kupffer and NK cells (Supplementary Figure 3B). For kidney, colon, prostate tumors, pancreatic cells from T2D, PBMC and Airways epithelium from SARS-CoV-2 patients's datasets and the annotations were used as provided in the corresponding publications (see data availability section).

\section{SPINT2 expression in TCGA tumors data}

TPM normalized counts from tumor samples in TCGA were downloaded (https://www.cancer.gov/tcga). Our analysis was restricted to tissue and sample matched tumor and normal samples only. Difference in average expression was estimated using Wilcoxon Test with Holm correction.

\section{Inference of Transcription Binding Sites}

A footprinting analysis was carried out using the TOBIAS pipeline ${ }^{69}$ with a default parameters setting of MACS -nomodel -shift -100 -extsize 200 -broad. Then, we extracted the inferred Transcription Factor Binding Sites (TFBS) for those TF with activities found to be positively correlated to both SPINT2 and TMPRSS2 using the single cell RNA-seq data. TFBS were visualised using the PlotTracks TOBIAS function and the network was built in Cytoscape ${ }^{70}$. Edges in the network represent TF binding scores.

\section{Data and script availability}

For this work we used the following datasets available in public repositories: scRNA-seq profiles of Calu-3 and $\mathrm{H} 1299$ cell lines ${ }^{22}$; scRNA-seq from ileum derived organoids ${ }^{18}$; translatome and proteome quantifications of Caco-2 cell line ${ }^{13}$; bulk RNA-seq profiles of lung samples of COVID-19 deceased patient autopsies ${ }^{44}$; Peripheral Blood Mononuclear Cells ${ }^{40}$; Nasopharynx samples from COVID-19 
patients ${ }^{38}$; Human Cell Landscape 25; The Cancer Genome Atlas . Tumor scRNA-seq datasets: Human Hepatocellular Carcinoma ${ }^{51}$, renal Clear Cell Carcinoma ${ }^{50}$, Lung Adenocarcinoma ${ }^{53}$, Colon Adenocarcinoma ${ }^{52}$, pancreatic cells from Diabetes Type 2 patients ${ }^{54}$; ATAC-seq data from Human intestinal organoids ${ }^{17}$.

All the codes for the data processing and analysis are provided in the following GitHub repository: https://github.com/hdsu-bioquant/covid19-comorbidity.

\section{Acknowledgments}

CRA acknowledges funding through the Deutsche Forschungsgemeinschaft (DFG, German Research Foundation) - Project-ID 272983813 - TRR 179. SB and CH acknowledge funding through the DFG program "Identification of the molecular origins of comorbidity in COVID-19 patients" (Project-ID 458633366).

\section{Figures captions}

Figure 1: Coregulation of SPINT2 and TMPRSS2. A. Peaks associated to open chromatin sites in regions containing the SPINT2 and TMPRSS2 genes (+/- 5kb of the coding regions) using ATAC-seq data from Human Intestinal Organoids. TF Binding Sites (TFBS) are scored based on the height and width of the peaks and the similarity of the sequences in the peaks to TF motifs. B. Correlation values of TF activities to SPINT2 and TMPRSS2 gene expression levels using scRNA-seq data from ileum derived organoids. Labeled TFs in $(A)$ and $(B)$ correspond to the intersection of inferred TFs bound to TFBS in (A) and those with positive correlation of TF activities to both SPINT2 and TMPRSS2 in (B), top right quadrant. C. Model for SPINT2 and TMPRSS2 coregulation. Edges from TFs (blue) to SPINT2 and TMPRSS2 (salmon) represent inferred binding shown in (A), $(B)$ and the edge width reflects TF binding scores. The blunt red arrow represents the inhibition of TMPRSS2 enzymatic activity by SPINT2. D. Correlation of SPINT2 and TMPRSS2 across tissues using bulk RNA-seq from GTEx. The additional red point labeled as nasopharynx represents the correlation of an independent dataset from nasopharynx tissue of COVID-19 patients. E. Gene expression of ACE2, SPINT2 and TMPRSS2 across cell types using HCL scRNA-seq data.

Figure 2: Permissivity genes are associated to viral related processes and correlated to viral expression. A. Permissivity signature genes ( $z$ ) were found using differential gene expression analysis (DEA) between Calu-3 and H1299 permissive and non-permissive cell lines. Similarly, 
infection signatures for both cell lines were calculated independently $(x+y)$ and filtered out from the permissive signature $(z)$ resulting in $\left(i^{\prime}\right)$. B. Permissive genes ranked by importance in predicting the cumulative sum of gene expression of viral genes. Only the top 10 ranked genes are labeled with names. Top $25 \%$ genes are shown in color and SPINT2 is highlighted in red. The inset plot show the number of genes in the intersection with each geneset. See the main text for the description of each gene set. C. Pathway Enrichment Analysis of the top $25 \%$ ranked genes. D. Ranking of cells in $\mathrm{HCL}$ using the permissivity scores based on the same genes as in (B) and (C). E. Correlation to viral proteins ( $\mathrm{S}$ and $\mathrm{N}$ genes) in the translatome data of Caco-2 cells. Gene names are coloured as in the pie chart in $(B)$.

Figure 3: SPINT2 knockdown in Calu-3 cell lines. A. Relative expression of SPINT2 normalized to the housekeeping gene TBP in wild type Calu-3, scrambled-treated and SPINT2 KD Calu-3 cells. B. Infection was detected by indirect immunofluorescence and percentage of infected cells was quantified C. Representative immunofluorescence images of wild type, scrambled control and SPINT2 $K D$ Calu-3 cells for mock-infected and SARS-CoV-2 infected conditions. D. The change in TMPRSS2 expression level was detected by qRT-PCT in WT, scrambled control and SPINT2 KD Calu-3 cells.

Figure 4: SPINT2 expression is associated with disease severity. A. Spearman correlation values of SPRGs to SARS-CoV-2 viral reads in scRNA-seq of nasopharynx samples from COVID-19 patients. Top 20 most correlated SPRGs are labeled and SPINT2 is highlighted in green. B. SPINT2, ACE2 and TMPRSS2 gene expression in severe and mild cases COVID-19 patients using datasets from Chua RL et al, 2020 C. Same as in (B) but using the Wilk A et al, 2020 data on Peripheral Blood Mononuclear Cells derived from COVID-19 patients

Figure 5: SPINT2 is down-regulated in tumors and diabetic pancreatic cells. Dot plots showing gene expression and percentage of cells expressing SPINT2, ACE2 and TMPRSS2 in tumors and pancreatic alpha cells. The selected cell type for each tumor type is indicated in each case. Lung Adenocarcinoma (LUAD), renal Clear Cell Carcinoma ( $r C C C)$, Hepatocyte Cell Carcinoma (HCC), Colon Adenocarcinoma (COAD), Type 2 diabetes (T2D).

Supplementary Figure 1. SPINT2 is negatively correlated to SARS-CoV-2 viral expression. A. Correlation of SPINT2 to the cumulative sum of normalized expression values of SARS-CoV-2 genes in Calu-3 cells. B. Correlation of SPINT2 to S viral protein translation rates in Caco-2 cells.

Supplementary Figure 2. SPINT2 expression in patients's datasets. ACE2, SPINT2 and TMPRSS2 expression in Chua RL et al, 2020 (A) and Blish C et al, 2020 (B) scRNA-seq datasets. SPINT2 expressing cell types shown in the main text are highlighted in red for both datasets. $\mathbf{C}$. Correlation of ACE2, SPINT2 and TMPRSS2 to viral proteins using bulk RNA-seq from COVID-19 deceased patients in Desai $\mathrm{N}$ et al, 2020.

Supplementary Figure 3. Analysis of SARS-CoV-2 associated comorbidity. A. Normalized gene expression (TPM) of SPINT2 in different cancers. P-values from Wilcoxon Test. B. Profiling of liver cells from hepatic tumors using cell type markers as described in Aizarani $\mathrm{N}$ et al, 2019, see methods. 
C. SPINT2 gene expression in pancreatic cells from diabetic patients. Hepatocytes and pancreatic alpha cells clusters highlighted in red represent cells for which gene expression profiles are shown in Figure 5.

\section{References}

1. Wrapp, D. et al. Cryo-EM structure of the 2019-nCoV spike in the prefusion conformation. Science 367, 1260-1263 (2020).

2. Bestle, D. et al. TMPRSS2 and furin are both essential for proteolytic activation of SARS-CoV-2 in human airway cells. Life Sci. Alliance 3, e202000786 (2020).

3. Hoffmann, M. et al. SARS-CoV-2 cell entry depends on ACE2 and TMPRSS2 and is blocked by a clinically proven protease inhibitor. Cell 181, 271-280.e8 (2020).

4. Zang, R. et al. TMPRSS2 and TMPRSS4 promote SARS-CoV-2 infection of human small intestinal enterocytes. Sci. Immunol. 5, eabc3582 (2020).

5. Hoffmann, M. et al. Nafamostat mesylate blocks activation of SARS-CoV-2: New treatment option for COVID-19. Antimicrobial agents and chemotherapy vol. 64 (2020).

6. Bojkova, D. et al. Aprotinin inhibits SARS-CoV-2 replication. Cells 9, 2377 (2020).

7. Asselta, R., Paraboschi, E. M., Mantovani, A. \& Duga, S. ACE2 and TMPRSS2 variants and expression as candidates to sex and country differences in COVID-19 severity in Italy. Aging (Albany NY) 12, 10087-10098 (2020).

8. Hou, Y. et al. New insights into genetic susceptibility of COVID-19: an ACE2 and TMPRSS2 polymorphism analysis. BMC Med. 18, 216 (2020).

9. Lucas, J. M. et al. The androgen-regulated protease TMPRSS2 activates a proteolytic cascade involving components of the tumor microenvironment and promotes prostate cancer metastasis. Cancer Discov. 4, 1310-1325 (2014).

10. Steinmetzer, T. \& Hardes, K. The antiviral potential of host protease inhibitors. in Activation of Viruses by Host Proteases 279-325 (Springer International Publishing, 2018).

11. Straus, M. R., Kinder, J. T., Segall, M., Dutch, R. E. \& Whittaker, G. R. SPINT2 inhibits proteases involved in activation of both influenza viruses and metapneumoviruses. Virology 543, 43-53 
(2020).

12. Ko, C.-J. et al. Inhibition of TMPRSS2 by HAI-2 reduces prostate cancer cell invasion and metastasis. Oncogene 39, 5950-5963 (2020).

13. Bojkova, D. et al. Proteomics of SARS-CoV-2-infected host cells reveals therapy targets. Nature 583, 469-472 (2020).

14. Roversi, F. M., Olalla Saad, S. T. \& Machado-Neto, J. A. Serine peptidase inhibitor Kunitz type 2 (SPINT2) in cancer development and progression. Biomed. Pharmacother. 101, 278-286 (2018).

15. Danielsen, E. T. et al. Intestinal regulation of suppression of tumorigenicity 14 (ST14) and serine peptidase inhibitor, Kunitz type -1 (SPINT1) by transcription factor CDX2. Sci. Rep. 8, (2018).

16. Friis, S. et al. The protease inhibitor HAl-2, but not HAI-1, regulates matriptase activation and shedding through prostasin. J. Biol. Chem. 289, 22319-22332 (2014).

17. Yin, S. et al. Functional genomics analysis of human colon organoids identifies key transcription factors. Physiol. Genomics 52, 234-244 (2020).

18. Triana, S. et al. Single-cell analyses reveal SARS-CoV-2 interference with intrinsic immune response in the human gut. bioRxiv (2020) doi:10.1101/2020.10.21.348854.

19. Van de Sande, B. et al. A scalable SCENIC workflow for single-cell gene regulatory network analysis. Nat. Protoc. 15, 2247-2276 (2020).

20. Patra, T. et al. SARS-CoV-2 spike protein promotes IL-6 trans-signaling by activation of angiotensin II receptor signaling in epithelial cells. PLoS Pathog. 16, e1009128 (2020).

21. Karki, R. et al. COVID-19 cytokines and the hyperactive immune response: Synergism of TNF- $\alpha$ and IFN- $\gamma$ in triggering inflammation, tissue damage, and death. bioRxiv (2020) doi:10.1101/2020.10.29.361048.

22. Emanuel, W. et al. Bulk and single-cell gene expression profiling of SARS-CoV-2 infected human cell lines identifies molecular targets for therapeutic intervention. bioRxiv (2020) doi:10.1101/2020.05.05.079194

23. Ng, A. Y.-N. et al. Inactivation of the transcription factor Elf3 in mice results in dysmorphogenesis and altered differentiation of intestinal epithelium. Gastroenterology 122, 1455-1466 (2002).

24. Sengez, B. et al. The Transcription Factor Elf3 Is Essential for a Successful Mesenchymal to Epithelial Transition. Cells 8, (2019).

25. Han, X. et al. Construction of a human cell landscape at single-cell level. Nature 581, 303-309 
(2020).

26. Gordon, D. E. et al. A SARS-CoV-2-human protein-protein interaction map reveals drug targets and potential drug-repurposing. bioRxivorg (2020) doi:10.1101/2020.03.22.002386.

27. Bausch-Fluck, D. et al. A mass spectrometric-derived cell surface protein atlas. PLoS One 10, e0121314 (2015).

28. Efremova, M., Vento-Tormo, M., Teichmann, S. A. \& Vento-Tormo, R. CellPhoneDB: inferring cell-cell communication from combined expression of multi-subunit ligand-receptor complexes.

Nat. Protoc. 15, 1484-1506 (2020).

29. Blanco-Melo, D. et al. SARS-CoV-2 launches a unique transcriptional signature from in vitro, ex vivo, and in vivo systems. bioRxiv (2020) doi:10.1101/2020.03.24.004655.

30. Meller, A., Coombs, D. \& Shalgi, R. Fluorescent polysome profiling reveals stress-mediated regulation of HSPA14-ribosome interactions. Cold Spring Harbor Laboratory 860833 (2020) doi:10.1101/860833.

31. Lamers, M. M. et al. SARS-CoV-2 productively infects human gut enterocytes. Science $\mathbf{3 6 9}$, 50-54 (2020)

32. Mulay, A. et al. SARS-CoV-2 infection of primary human lung epithelium for COVID-19 modeling and drug discovery. bioRxivorg (2020) doi:10.1101/2020.06.29.174623.

33. Lodermeyer, V. et al. The antiviral activity of the cellular glycoprotein LGALS3BP/90K is species specific. J. Virol. 92, (2018).

34. Hepojoki, J. et al. Acute hantavirus infection induces galectin-3-binding protein. J. Gen. Virol. 95, 2356-2364 (2014).

35. Hong, C.-S. et al. Gal-3BP Negatively Regulates NF-KB Signaling by Inhibiting the Activation of TAK1. Front. Immunol. 10, 1760 (2019).

36. Stakaitytè, G. et al. The cellular chloride channels CLIC1 and CLIC4 contribute to virus-mediated cell motility. J. Biol. Chem. 293, 4582-4590 (2018).

37. Wyżewski, Z., Gregorczyk, K. P., Szczepanowska, J. \& Szulc-Dabrowska, L. Functional role of Hsp60 as a positive regulator of human viral infection progression. Acta Virol. 62, 33-40 (2018).

38. Chua, R. L. et al. COVID-19 severity correlates with airway epithelium-immune cell interactions identified by single-cell analysis. Nat. Biotechnol. 38, 970-979 (2020).

39. Zhu, N. et al. Morphogenesis and cytopathic effect of SARS-CoV-2 infection in human airway 
epithelial cells. Nat. Commun. 11, 3910 (2020).

40. Wilk, A. J. et al. A single-cell atlas of the peripheral immune response in patients with severe COVID-19. Nat. Med. 26, 1070-1076 (2020).

41. Desai, N. et al. Temporal and spatial heterogeneity of host response to SARS-CoV-2 pulmonary infection. medRxiv (2020) doi:10.1101/2020.07.30.20165241.

42. Cheng, Y. et al. Kidney disease is associated with in-hospital death of patients with COVID-19. Kidney Int. 97, 829-838 (2020).

43. Jothimani, D., Venugopal, R., Abedin, M. F., Kaliamoorthy, I. \& Rela, M. COVID-19 and the liver. J. Hepatol. 73, 1231-1240 (2020).

44. Sanyaolu, A. et al. Comorbidity and its Impact on Patients with COVID-19. SN Compr Clin Med $1-8(2020)$.

45. Guan, W.-J. et al. Comorbidity and its impact on 1590 patients with Covid-19 in China: A Nationwide Analysis. Eur. Respir. J. (2020) doi:10.1183/13993003.00547-2020.

46. Guan, W.-J., Liang, W.-H., He, J.-X. \& Zhong, N.-S. Cardiovascular comorbidity and its impact on patients with COVID-19. Eur. Respir. J. 55, (2020).

47. Fukushima, T. et al. Aberrant methylation and silencing of the SPINT2 gene in high-grade gliomas. Cancer Sci. 109, 2970-2979 (2018).

48. Dong, W., Chen, X., Xie, J., Sun, P. \& Wu, Y. Epigenetic inactivation and tumor suppressor activity of HAI-2/SPINT2 in gastric cancer. Int. J. Cancer 127, 1526-1534 (2010).

49. Qu, Y., Dang, S. \& Hou, P. Gene methylation in gastric cancer. Clin. Chim. Acta 424, 53-65 (2013).

50. Young, M. D. et al. Single-cell transcriptomes from human kidneys reveal the cellular identity of renal tumors. Science 361, 594-599 (2018).

51. Lu, Y. et al. A Single-Cell Atlas of the Multicellular Ecosystem of Primary and Metastatic Hepatocellular Carcinoma. (2020) doi:10.2139/ssrn.3624436.

52. Li, H. et al. Reference component analysis of single-cell transcriptomes elucidates cellular heterogeneity in human colorectal tumors. Nat. Genet. 49, 708-718 (2017).

53. Laughney, A. M. et al. Regenerative lineages and immune-mediated pruning in lung cancer metastasis. Nat. Med. 26, 259-269 (2020).

54. Segerstolpe, $\AA$. et al. Single-cell transcriptome profiling of human pancreatic islets in health and 
type 2 diabetes. Cell Metab. 24, 593-607 (2016).

55. Seget-Trzensiok, K. et al. USP28 and SPINT2 mediate cell cycle arrest after whole genome doubling. (2020) doi:10.1101/2020.09.18.303834.

56. Poddar, N. K., Maurya, S. K. \& Saxena, V. Role of Serine Proteases and Inhibitors in Cancer. in Proteases in Physiology and Pathology 257-287 (Springer Singapore, 2017).

57. Finotti, P. The role played by serine proteases in the development and worsening of vascular complications in type 1 diabetes mellitus. Curr. Diabetes Rev. 2, 295-305 (2006).

58. Sharony, R. et al. Protein targets of inflammatory serine proteases and cardiovascular disease. J. Inflamm. (Lond.) 7, 45 (2010).

59. Martin, C. E. \& List, K. Cell surface-anchored serine proteases in cancer progression and metastasis. Cancer Metastasis Rev. 38, 357-387 (2019).

60. Goel, A. \& Chauhan, S. S. Role of proteases in tumor invasion and metastasis. Indian J. Exp. Biol. 35, 553-564 (1997).

61. Parr, C., Sanders, A. J. \& Jiang, W. G. Hepatocyte growth factor activation inhibitors - therapeutic potential in cancer. Anticancer Agents Med. Chem. 10, 47-57 (2010).

62. Yang, J.-K., Lin, S.-S., Ji, X.-J. \& Guo, L.-M. Binding of SARS coronavirus to its receptor damages islets and causes acute diabetes. Acta Diabetol. 47, 193-199 (2010).

63. Mason, S. D. \& Joyce, J. A. Proteolytic networks in cancer. Trends Cell Biol. 21, 228-237 (2011).

64. Yang, X. et al. A public genome-scale lentiviral expression library of human ORFs. Nat. Methods 8, 659-661 (2011).

65. Hao, Y. et al. Integrated analysis of multimodal single-cell data. Cold Spring Harbor Laboratory 2020.10.12.335331 (2020) doi:10.1101/2020.10.12.335331.

66. Kuleshov, M. V. et al. Enrichr: a comprehensive gene set enrichment analysis web server 2016 update. Nucleic Acids Res. 44, W90-7 (2016).

67. Li, W. V. \& Li, J. J. An accurate and robust imputation method sclmpute for single-cell RNA-seq data. Nat. Commun. 9, 997 (2018).

68. Aizarani, N. et al. A human liver cell atlas reveals heterogeneity and epithelial progenitors. Nature 572, 199-204 (2019).

69. Bentsen, M. et al. ATAC-seq footprinting unravels kinetics of transcription factor binding during zygotic genome activation. Nat. Commun. 11, 4267 (2020). 
bioRxiv preprint doi: https://doi.org/10.1101/2020.12.28.424029; this version posted December 28, 2020. The copyright holder for this

preprint (which was not certified by peer review) is the author/funder, who has granted bioRxiv a license to display the preprint in perpetuity. It is made available under aCC-BY-NC-ND 4.0 International license.

70. Shannon, P. et al. Cytoscape: a software environment for integrated models of biomolecular interaction networks. Genome Res. 13, 2498-2504 (2003). 


\section{Figure 1}

A

chr 19: 38,239,035 - 38,297,615 (+) [58580 bp]

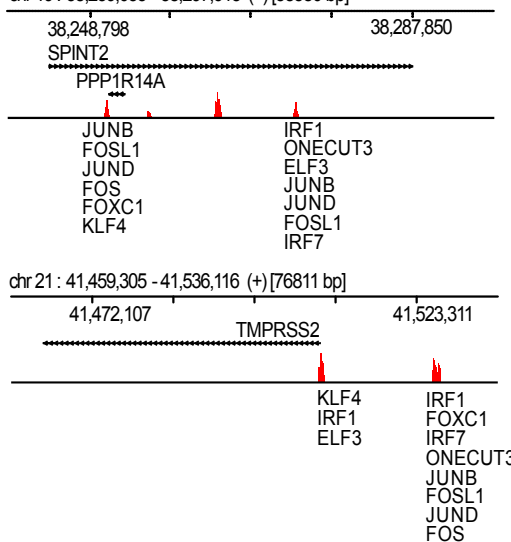

C FOS JUND

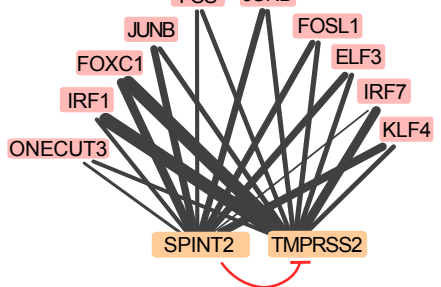

B

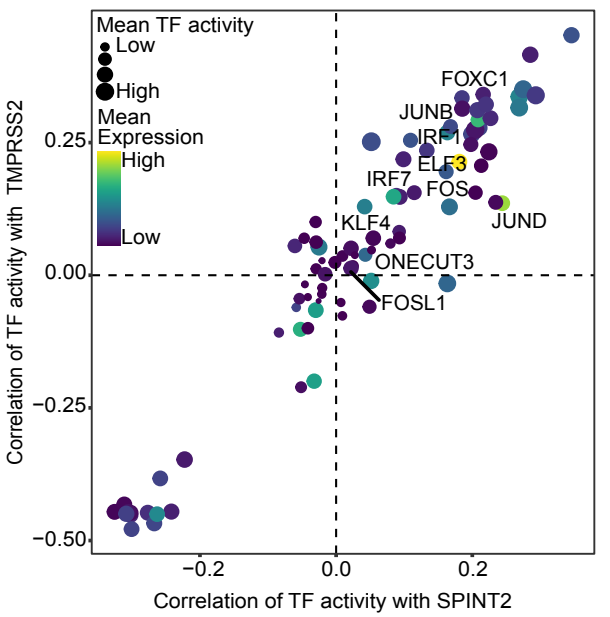

D

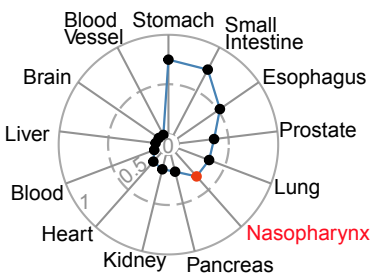

E

Mean

Expression
High

0 - Proximal tubule progenitor

-Gastric endocrine cell

O-Stratified epithelial cell

- Loop of Henle

O O-Goblet cell

○ ० - Enterocyte

O 0 -AT2 cell

- - Enterocyte progenitor

$\%$ cells

expressed

10

20

$\bigcap^{30}$

- Epithelial cell

○ - Epithelial cell

- 0 -Gastric chief cell

- Dendritic cell

- Endothelial cell

- M2 Macrophage

Macrophage

- Sinusoidal endothelial cell Endothelial cell (APC)

-Smooth muscle cell

Fibroblast

Endothelial cell

-Stromal cell

Ventricle cardiomyocyte hESC

O ○ Hepatocyte/Endodermal cell Kidney intercalated cell 


\section{gure 2}
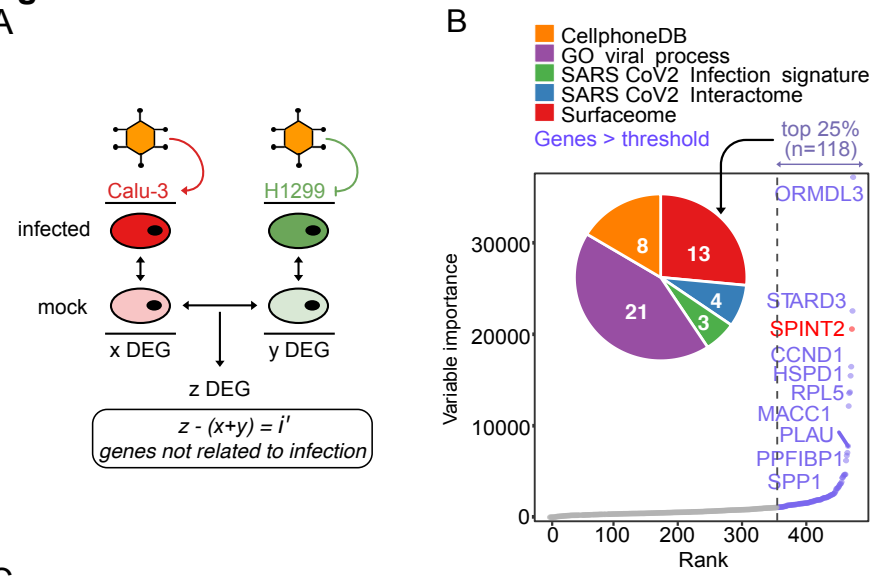

C

B
D Stratified epithelial cell Basal cell oop of Henle Epithelial cell AT2 cell Enterocyte te progenitor Enterocyte progenitor
Chondrocyte Gastric endocrine cell Proximal tubule progenitor Eithelial cell M2 Macrophage intermc bud cell Intermediated cell

hyroid follicular cell Mesothelial cell Hepatocyte/Endodermal cell Endothelial cell Endothelial cell Macrophage Endothelial cell (APC) Kidney intercalated cell Mibroblast Gastric chief cell Smooth muscle cell Pancreas exocrine cell

\section{-}

E

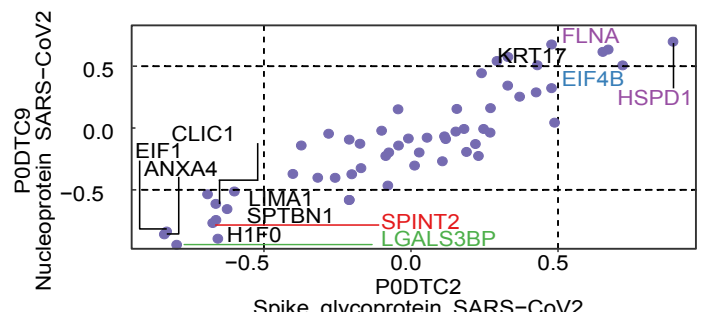

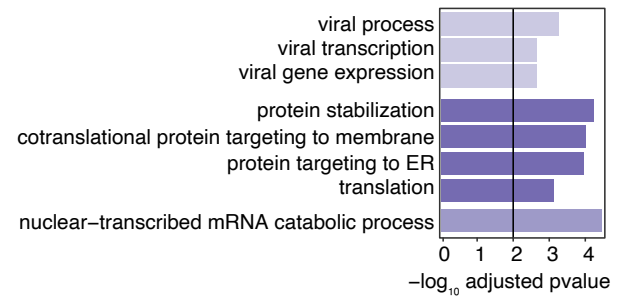




\section{Figure 3}

A

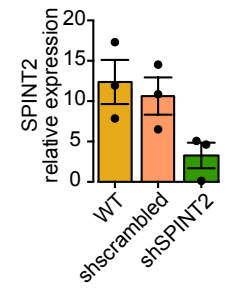

B

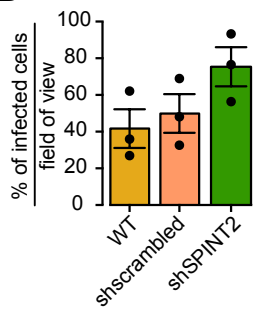

D

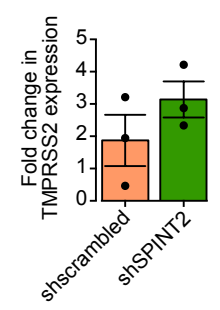

C
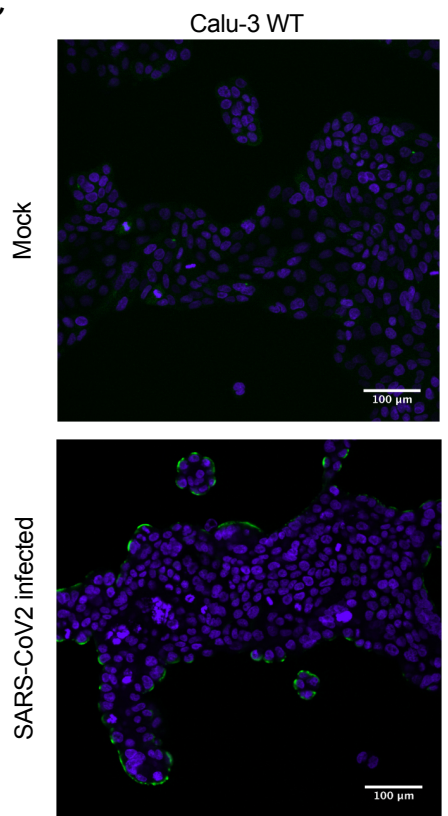

Calu-3 scrambled

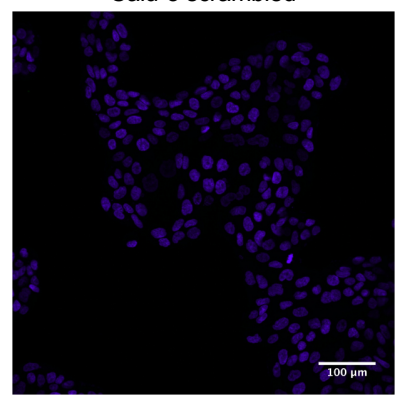

Calu-3 SPINT2 KD

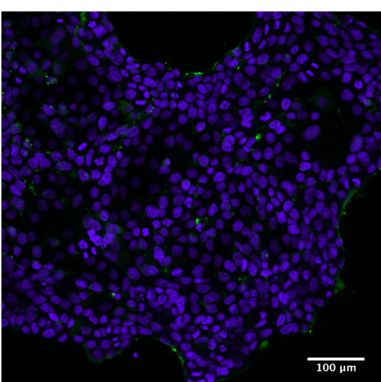

$\overline{100 \mu \mathrm{m}}$

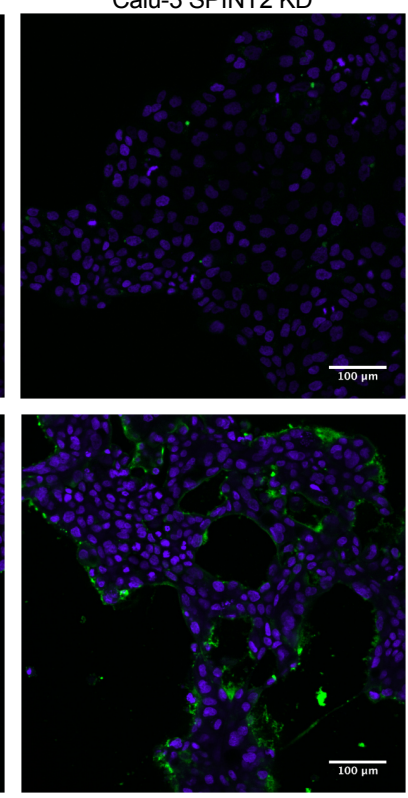




\section{Figure 4}

A

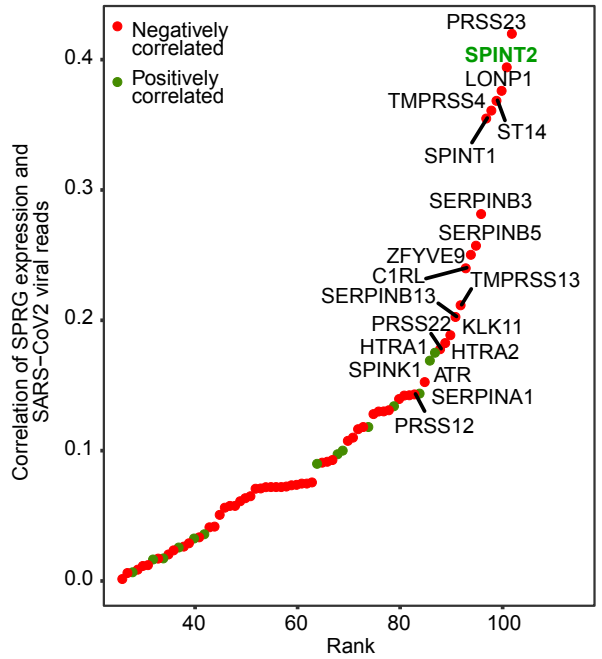

B

Chua RL. et.al, 2020 (Secretory cells)

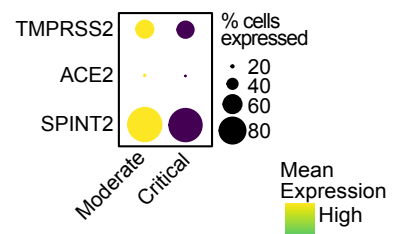

C Wilk A. et.al., 2020 (DC, pDC \& Eosinophils)

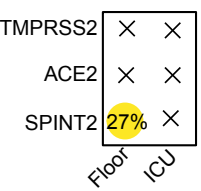




\section{Figure 5}

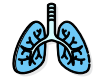

LUAD

(Epithelial cells)

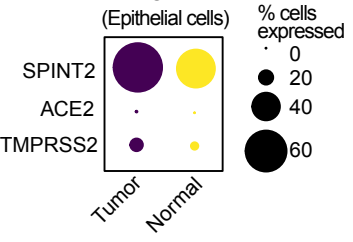

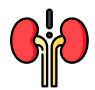

rCCC

(Endothelial cells) \% cells

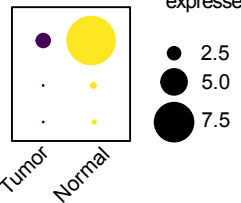

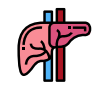

$\mathrm{HCC}$

(Hepatocytes)

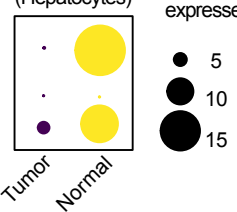

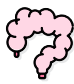

COAD

(Epithelial cells) \% cells

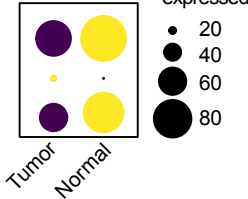

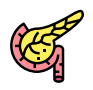

T2D

(Alpha cells)

$\%$ cells

Mean

expressed Expression

0 - $25 \quad$ High

- 50

$0^{75}$

100

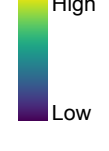



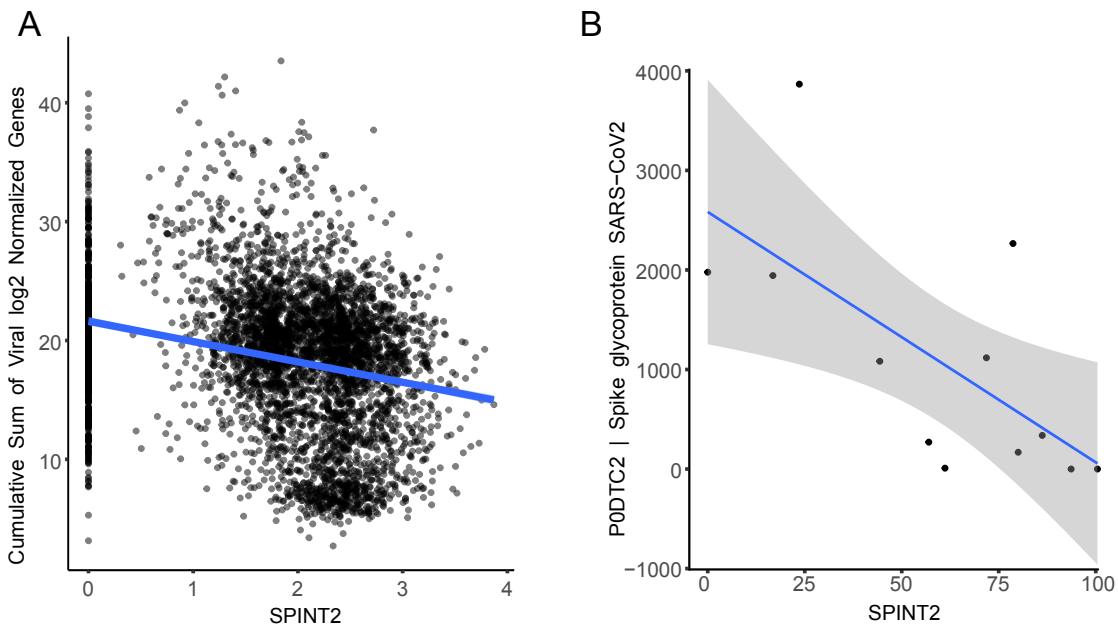


\section{Supplementary Figure 2}

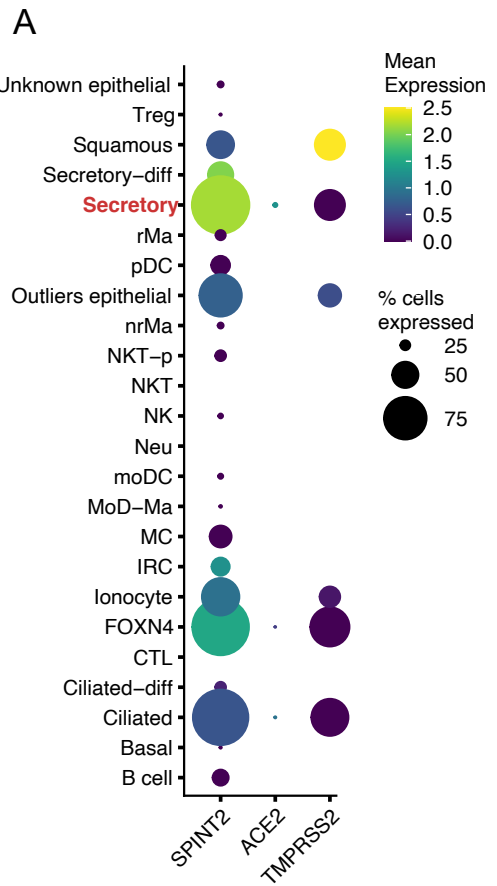

B

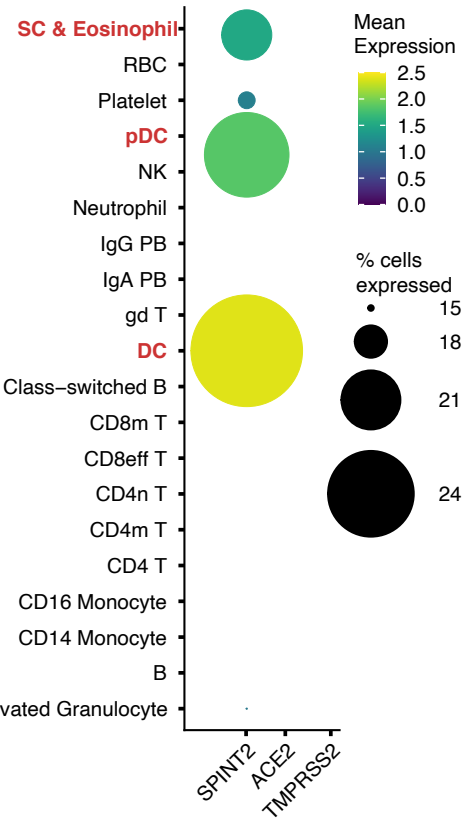

C

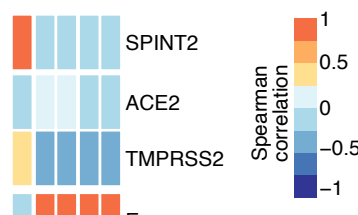




\section{Supplementary Figure 3}

A
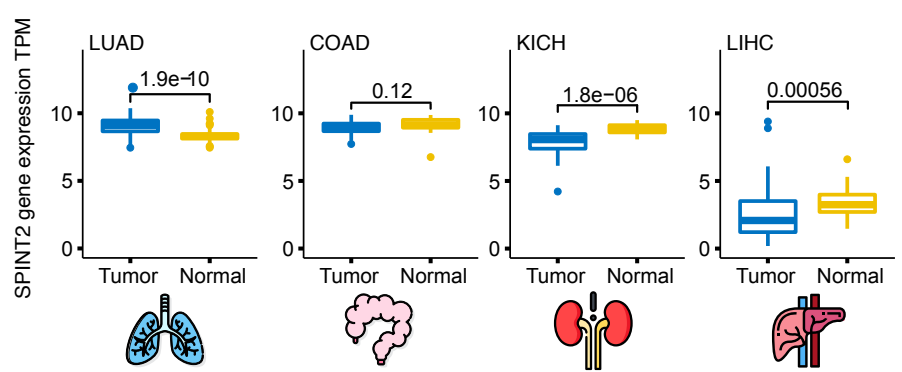

B

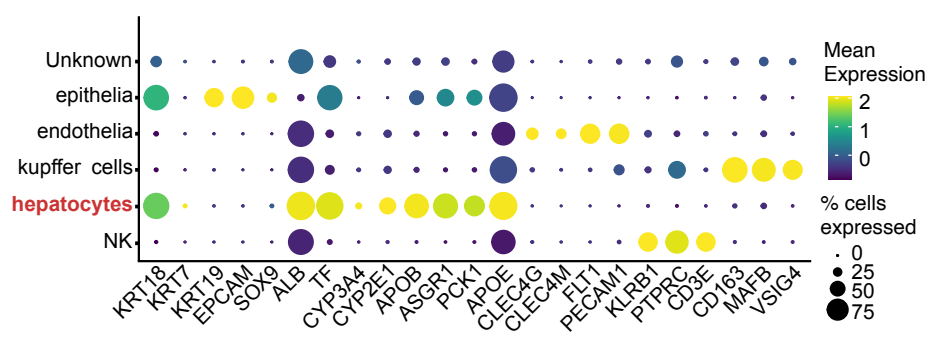

C

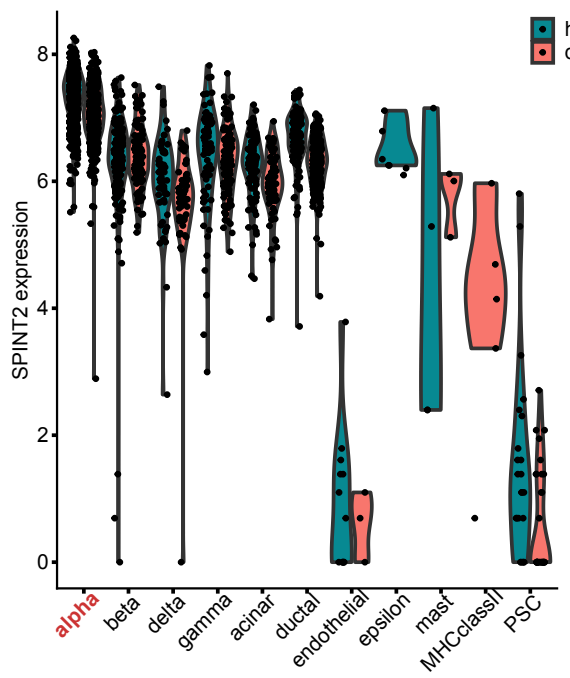

healthy/control disease 